\title{
On Non-Associative Rings
}

\author{
Ida Kurnia Waliyanti ${ }^{1,2, *}$, Indah Emilia Wijayanti ${ }^{1}$, M. Farchani Rosyid $^{3}$ \\ ${ }^{1}$ Department of Mathematics, Universitas Gadjah Mada, Yogyakarta, 55281, Indonesia \\ ${ }^{2}$ Department of Mathematics Education, Universitas Khairun, Ternate, 97719, Indonesia \\ ${ }^{3}$ Department of Physics, Universitas Gadjah Mada, Yogyakarta, 55281, Indonesia
}

Cite This Paper in the following Citation Styles

(a): [1] Ida KurniaWaliyanti, Indah Emilia Wijayanti, M. Farchani Rosyid, "On Non-Associative Rings," Mathematics and Statistics, Vol.9, No.2, pp. 172-177, 2021. DOI: 10.13189/ms.2021.090212.

(b): Ida KurniaWaliyanti, Indah Emilia Wijayanti, M. Farchani Rosyid, (2021). On Non-Associative Rings. Mathematics and Statistics, 9(2), 172-177. DOI: 10.13189/ms.2021.090212.

Copyright $@ 2021$ by authors, all rights reserved. Authors agree that this article remains permanently open access under the terms of the Creative Commons Attribution License 4.0 International License

\begin{abstract}
Jordan ring is one example of the non-associative rings. We can construct a Jordan ring from an associative ring by defining the Jordan product. In this paper, we discuss the properties of non-associative rings by studying the properties of the Jordan rings. All of the ideals of a non-associative ring $R$ are non-associative, except the ideal generated by the associator in $R$. Hence, a quotient ring $R /\langle X\rangle$ can be constructed, where $\langle X\rangle$ is the ideal generated by associators in $R$. The fundamental theorem of the homomorphism ring can be applied to the non-associative rings. By a little modification, we can find that $R /\langle X\rangle$ is isomorphic to $S /\langle Y\rangle$. Furthermore, we define a module over a non-associative ring and investigate its properties. We also give some examples of such modules. We show if $M$ is a module over a non-associative ring $R$, then $M$ is also a module over $R /\langle X\rangle$ if $\langle X\rangle$ is contained in the annihilator of $R$. Moreover, we define the tensor product of modules over a non-associative ring. The tensor product of the modules over a non-associative ring is commutative and associative up to isomorphism but not element by element.
\end{abstract}

Keywords Non-Associative Ring, Jordan Ring, Ideal, Module, Tensor Product

\section{Introduction}

In the development of the ring theory, there has been much discussion about the associative ring. The term ring usually refers to an associative ring unless we mention it more precisely.

Several researchers have investigated non-associative rings, for examples Shah [1] and Razzaque [2]. Reference [1] explains a survey of non-associative rings. It is mentioned here that some examples of non-associative rings are octonions, Lie rings, alternative rings, Jordan rings, and loop rings. The ring $\mathbb{R}^{3}$ with the addition $(+)$ and cross-product $(\times)$, i.e. $\left(\mathbb{R}^{3},+, \times\right)$ is an example of Lie ring.

In this paper, we investigate the Jordan ring which is nonassociative, commutative, and preserves the Jordan identity. Jordan ring also can be constructed from any ring $A$ [1]. If $A$ is commutative, then a Jordan ring formed is associative, but the converse is not always true. The explanation of such property and examples will be given in the second section.

After explaining the Jordan ring, we define the ideals of the non-associative ring. We will also construct a quotient ring on a non-associative ring. Therefore, we find the associative ideal in a non-associative ring. We also discuss non-associative ring homomorphisms.

In the next section we define the module over a nonassociative ring. However, Carotenuto [3, 4] has given a different definition and called it the Jordan module. This definition began from the understanding of Jordan algebra. The Jordan algebra has a structure similar to the Jordan ring, which is commutative, non-associative, and satisfies the Jordan identity. The set of self-adjoint real matrices with Jordan product as multiplication is an example of Jordan algebras. From here, Carotenuto [3, 4] defined a Jordan module.

Definition 1.1. Let $\mathbf{J}$ be a Jordan algebra and $V$ be a real vector space together with two linear mappings, i.e.

$$
\mathbf{J} \otimes V \rightarrow V, \quad j \otimes v \mapsto j v
$$

and

$$
V \otimes \mathbf{J} \rightarrow V, \quad v \otimes j \mapsto v j,
$$


such that $\mathbf{J} \oplus V$, endowed with the product

$$
(j, v)\left(j^{\prime}, v^{\prime}\right)=\left(j j^{\prime}, j v^{\prime}+v^{\prime} j\right)
$$

is a Jordan algebra, and $V$ is called a Jordan module.

Here are some examples of Jordan module:

Example 1.2. Jordan algebra is a Jordan module over itself.

Example 1.3. Let $M$ be an abelian group and $R$ an associative ring. Algebra $R_{J}$ is Jordan algebra that formed from $R$. Module $M$ is an $R_{J}$-module is an example of the Jordan module.

A Jordan module is one example of the modules over a nonassociative ring, as defined in 2.10 .

In our work, we observe modules over a non-associative ring in a more general point of view. Moreover, we conduct the tensor product of modules over the non-associative ring $R_{J}$.

\section{Result and Discuss}

\subsection{Non-Associative Rings}

Ring $R$ is a non-associative ring if it does not require associative properties in the multiplication operation. Several examples have been mentioned in the first section. One of them is Jordan ring. In the beginning of this section we discuss the construction of the Jordan ring and some properties. A Jordan ring is a generalization of Jordan algebra. Reference [4] defines the Jordan algebras as a vector space together with a bilinear product that commutes and satisfies the Jordan identity.

Definition 2.1. A ring $A$ which satisfies the following two axioms:

$$
\begin{aligned}
& \text { 1. } a b=b a, \\
& \text { 2. } a\left(b a^{2}\right)=(a b) a^{2} \text {, }
\end{aligned}
$$

for all $a, b \in A$, is a Jordan ring.

Reference [1] shows that Jordan ring can be constructed from any ring. For any ring $R$ one can define the Jordan product as follow for each $r_{1}, r_{2} \in R, r_{1} \circ r_{2}=r_{1} r_{2}+r_{2} r_{1}$. So we get a new ring under the addition and the Jordan product, denoted by $R_{J}$.

However, not all Jordan rings formed from any ring $R$ are non-associative. The following lemma will explain these properties.

Lemma 2.2. Let any commutative ring $R$, so $R_{J}$ is associative.

Proof. For any $r_{1}, r_{2}, r_{3} \in R$,

$$
\begin{aligned}
r_{1} \circ\left(r_{2} \circ r_{2}\right) & =r_{1} \circ\left(r_{2} r_{3}+r_{3} r_{2}\right) \\
& =r_{1}\left(r_{2} r_{3}+r_{3} r_{2}\right)+\left(r_{2} r_{3}+r_{3} r_{2}\right) r_{1} \\
& =r_{1} r_{2} r_{3}+r_{1} r_{3} r_{2}+r_{2} r_{3} r_{1}+r_{3} r_{2} r_{1} \\
& =r_{1} r_{2} r_{3}+r_{2} r_{1} r_{3}+r_{3} r_{1} r_{2}+r_{3} r_{2} r_{1} \\
& =\left(r_{1} r_{2}+r_{2} r_{1}\right) r_{3}+r_{3}\left(r_{1} r_{2}+r_{2} r_{1}\right) \\
& =\left(r_{1} \circ r_{2}\right) r_{3}+r_{3}\left(r_{1} \circ r_{2}\right) \\
& =\left(r_{1} \circ r_{2}\right) \circ r_{3} .
\end{aligned}
$$

Thus for any commutative ring $R$, we get Jordan ring $R_{J}$, which is associative.

Example 2.3. Consider a ring of real numbers $\mathbb{R}$ which is a commutative ring. So we get an associative ring $\mathbb{R}_{J}$.

If $R$ has a unit, then $R_{J}$ does not necessarily have a unit. For example, the ring $\mathbb{Z}$ has 1 as unit. The unit of $\mathbb{Z}_{J}$ is $\frac{1}{2}$, however $\frac{1}{2} \notin \mathbb{Z}_{J}$. So $\mathbb{Z}_{J}$ does not have a unit obviously. Next, given a non-commutative ring $M_{2 \times 2}(\mathbb{R})$, which has $\left(\begin{array}{ll}1 & 0 \\ 0 & 1\end{array}\right)$ as unit. We can construct a Jordan ring $M_{J_{2 \times 2}}$ which has unit, i.e. $\left(\begin{array}{cc}\frac{1}{2} & 0 \\ 0 & \frac{1}{2}\end{array}\right)$. It shows that there exists a non-associative ring which has a unit.

Lemma 2.2 states that every commutative ring $R$, will construct $R_{J}$ which is associative. However, the converse of this statement does not apply. The set of all quaternions, denoted by $\mathbb{H}$, is an example of a non-commutative ring having Jordan product, leading to the associative ring $\mathbb{H}_{J}$. This shows that not all non-commutative rings in which the Jordan product is defined lead to the non-associative rings.

Now, we have a non-associative ring $R$. Eventhough $R$ is non-associative, $\operatorname{End}_{R}(R)$ is associative. This is because for every $\lambda, \rho, \phi \in \operatorname{End}(R), \lambda \circ(\rho \circ \phi)=(\lambda \circ \rho) \circ \phi$. Since $\operatorname{End}_{R}(R)$ is associative, it is clear that $R$ is not isomorphic to $\operatorname{End}_{R}(R)$.

Now we will introduce ideal of the non-associative ring.

Definition 2.4. A subset $\emptyset \neq I$ in $R$ is an ideal of $R$ if

1. $(I,+)$ is subgroup of $R$,

2. $r I, I r \subseteq I$, for all $r \in R$.

By the definition, we conclude that all ideals in $R$ are nonassociative, except ideal that generated by $X=\{u(v w)-$ $(u v) w \mid u, v, w \in R\}$.

The left ideal generated by $X$ is

$\langle X\rangle_{l}=\left\{\sum_{i=1}^{n} r_{i}\left(u_{i}\left(v_{i} w_{i}\right)-\left(u_{i} v_{i}\right) w_{i}\right) \sum_{j=1}^{t} k_{j}\left(u_{j}^{\prime}\left(v_{j}^{\prime} w_{j}^{\prime}\right)-\right.\right.$ $\left.\left(u_{j}^{\prime} v_{j}^{\prime}\right) w_{j}^{\prime}\right) \mid n, k \in \mathbb{N}, k_{j} \in \mathbb{Z}, r_{i} \in R, u_{i}, v_{i}, w_{i}, u_{j}^{\prime}, v_{j}^{\prime}, w_{j}^{\prime} \in$ $R\}$

The right ideal generated by $X$ is $\langle X\rangle_{r}=\left\{\sum_{i=1}^{n}\left(u_{i}\left(v_{i} w_{i}\right)-\left(u_{i} v_{i}\right) w_{i}\right) r_{i} \sum_{j=1}^{t} k_{j}\left(u_{j}^{\prime}\left(v_{j}^{\prime} w_{j}^{\prime}\right)-\right.\right.$
$\left.\left(u_{j}^{\prime} v_{j}^{\prime}\right) w_{j}^{\prime}\right) \mid n, k \in \mathbb{N}, k_{j} \in \mathbb{Z}, r_{i} \in R, u_{i}, v_{i}, w_{i}, u_{j}^{\prime}, v_{j}^{\prime}, w_{j}^{\prime} \in$
$R\}$

The ideal generated by $X$ is $\langle X\rangle=\left\{\sum_{i=1}^{n} r_{i}\left(u_{i}\left(v_{i} w_{i}\right)-\left(u_{i} v_{i}\right) w_{i}\right) r_{i} \sum_{j=1}^{t} k_{j}\left(u_{j}^{\prime}\left(v_{j}^{\prime} w_{j}^{\prime}\right)-\right.\right.$
$\left.\left(u_{j}^{\prime} v_{j}^{\prime}\right) w_{j}^{\prime}\right) \mid n, k \in \mathbb{N}, k_{j} \in \mathbb{Z}, r_{i} \in R, u_{i}, v_{i}, w_{i}, u_{j}^{\prime}, v_{j}^{\prime}, w_{j}^{\prime} \in$
$R\}$

We can say that $X$ is a subset of $R$ whose elements are associator in $R$. The subset $X$ constructs a quotient ring, denoted by $R /\langle X\rangle$. This quotient ring $R /\langle X\rangle$ is associative. 
Lemma 2.5. Let $R$ be any non-associative ring and subset $X=\{u(v w)-(u v) w \mid u, v, w \in R\}$. The quotient ring $R /\langle X\rangle$ is associative.

Proof. It is sufficient show that $R /\langle X\rangle$ is associative. Let $\overline{r_{1}}, \overline{r_{2}}, \overline{r_{3}} \in R /\langle X\rangle$. We will show that $\overline{r_{1}}\left(\overline{r_{2} r_{3}}\right)=\left(\overline{r_{1} r_{2}}\right) \overline{r_{3}}$.

$$
\begin{aligned}
\overline{r_{1}}\left(\overline{r_{2}} \overline{r_{3}}\right) & =\overline{r_{1}}\left(\overline{r_{2} r_{3}}\right) \\
& =\overline{r_{1}\left(r_{2} r_{3}\right)}
\end{aligned}
$$

On the other hand

$$
\begin{aligned}
\left(\overline{r_{1}} \overline{r_{2}}\right) \overline{r_{3}} & =\left(\overline{r_{1} r_{2}}\right) \overline{r_{3}} \\
& =\overline{\left(r_{1} r_{2}\right) r_{3}}
\end{aligned}
$$

To make the associative valid we should that $\overline{r_{1}\left(r_{2} r_{3}\right)}=\overline{\left(r_{1} r_{2}\right) r_{3}}$. It means $r_{1}\left(r_{2} r_{3}\right)-\left(r_{1} r_{2}\right) r_{3} \in X$. It is clear since $X=\{u(v w-(u v) w \mid u, v, w \in R\}$.

Thus we can find a quotient ring $R /\langle X\rangle$, which is associative. The definition of non-associative ring homomorphism is following.

Definition 2.6. Let $R$ and $P$ be non-associative rings. The map $\alpha: R \rightarrow P$ is a ring homomorphism if it satisfies the following two axioms:

1. $\alpha\left(r_{1}+r_{2}\right)=\alpha\left(r_{1}\right)+\alpha\left(r_{2}\right)$

2. $\alpha\left(r_{1} r_{2}\right)=\alpha\left(r_{1}\right) \alpha\left(r_{2}\right)$.

In correlation with the fundamental theorem of ring homomorphism, the following properties are obtained.

Lemma 2.7. Let $R$ and $P$ be non-associative rings and $X=$ $\{u(v w)-(u v) w \mid u, v, w \in R\} \subset R$. If $\alpha: R \rightarrow P$ is a ring homomorphism and $\langle X\rangle \subseteq \operatorname{Ker}(\alpha)$, then there exists $\varphi$ such that

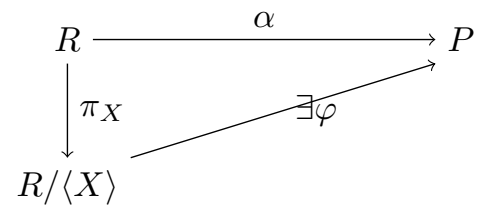

Proof. Define $\varphi: R /\langle X\rangle \rightarrow P$ by $\varphi(r+R /\langle X\rangle)=\alpha(r)$. For any $r_{1}+\langle X\rangle=r_{2}+\langle X\rangle \in R /\langle X\rangle$ it means $r_{1}-r_{2} \in\langle X\rangle$. We show that $\varphi\left(r_{1}+\langle X\rangle\right)=\varphi\left(r_{2}+\langle X\rangle\right)$

$$
\begin{aligned}
& \varphi\left(r_{1}+\langle X\rangle\right)=\alpha\left(r_{1}\right) \\
& \varphi\left(r_{2}+\langle X\rangle\right)=\alpha\left(r_{2}\right)
\end{aligned}
$$

so it remains only to show that $\alpha\left(r_{1}\right)=\alpha\left(r_{2}\right)$. Because $r_{1}-$ $r_{2} \in\langle X\rangle \subseteq \operatorname{ker}(\alpha)$, so

$$
\begin{aligned}
\alpha\left(r_{1}-r_{2}\right) & =0 \\
\alpha\left(r_{1}\right)-\alpha\left(r_{2}\right) & =0 \\
\alpha\left(r_{1}\right) & =\alpha\left(r_{2}\right)
\end{aligned}
$$

So $\varphi: R /\langle X\rangle \rightarrow P$ is well define. We show that $\alpha=\varphi \circ \pi_{X}$. For any $r \in R$

$$
\begin{aligned}
\left(\varphi \circ \pi_{X}\right)(r) & =\varphi\left(\pi_{X}(r)\right) \\
& =\varphi(r+\langle X\rangle) \\
& =\alpha(r) .
\end{aligned}
$$

In associative case, if $\alpha$ surjective and $\operatorname{ker}(\alpha)=\langle X\rangle$ then $R /\langle X\rangle \cong P$. But $R /\langle X\rangle$ is associative and $P$ is nonassociative, so it is clear that $R /\langle X\rangle \varsubsetneqq P$. This motivates us to develop the homomorphism theorem as follows:

Propotition 2.8. Let $R$ and $P$ be non-associative rings, $X=\{u(v w)-(u v) w \mid u, v, w \in R\}$ and $Y=$ $\left\{u^{\prime}\left(v^{\prime} w^{\prime}\right)-\left(u^{\prime} v^{\prime}\right) w^{\prime} \mid u^{\prime}, v^{\prime}, w^{\prime} \in P\right\}$. If $\alpha: R \rightarrow$ $P$ is a ring homomorphism and $\langle X\rangle \subseteq \operatorname{Ker}(\alpha)$ then there exists $\varphi$ such that $\pi_{Y} \circ \alpha=\varphi \circ \pi_{X}$.

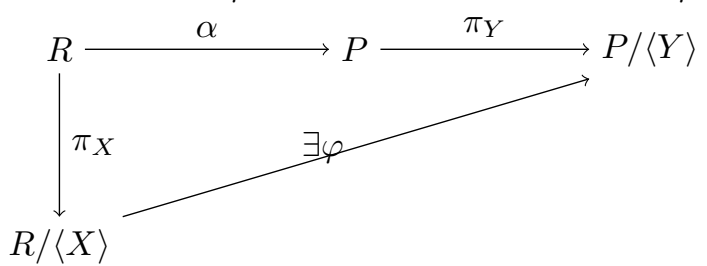

Proof. Define $\varphi: R /\langle X\rangle \rightarrow P /\langle Y\rangle$ with $\varphi(r+\langle X\rangle)=$ $\alpha(r)+\langle Y\rangle$. For any $r_{1}+\langle X\rangle=r_{2}+\langle X\rangle \in R /\langle X\rangle$ which means $r_{1}-r_{2} \in\langle X\rangle$, we will show that $\varphi\left(r_{1}+\langle X\rangle\right)=\varphi\left(r_{2}+\right.$ $\langle X\rangle)$. Because $\varphi\left(r_{1}+\langle X\rangle\right)=\alpha\left(r_{1}\right)+\langle Y\rangle$ and $\varphi\left(r_{2}+\langle X\rangle\right)=$ $\alpha\left(r_{2}\right)+\langle Y\rangle$ so it remains only to show that $\alpha\left(r_{1}\right)+\langle Y\rangle=$ $\alpha\left(r_{2}\right)+\langle Y\rangle$ or $\alpha\left(r_{1}\right)-\alpha\left(r_{2}\right) \in\langle Y\rangle$. Since $r_{1}-r_{2} \in\langle X\rangle \subseteq$ $\operatorname{ker}(\alpha)$, so

$$
\begin{aligned}
\left(\pi_{Y} \circ \alpha\right)\left(r_{1}-r_{2}\right) & =\pi_{Y}\left(\alpha\left(r_{1}-r_{2}\right)\right) \\
& =\alpha\left(r_{1}-r_{2}\right)+\langle Y\rangle \\
& =0+\langle Y\rangle \\
& =\langle Y\rangle .
\end{aligned}
$$

It means

$$
\begin{aligned}
\alpha\left(r_{1}-r_{2}\right) & \in\langle Y\rangle \\
\alpha\left(r_{1}\right)-\alpha\left(r_{2}\right) & \in\langle Y\rangle
\end{aligned}
$$

So $\alpha\left(r_{1}\right)+\langle Y\rangle=\alpha\left(r_{2}\right)+\langle Y\rangle$. Then $\varphi: R /\langle X\rangle \rightarrow P /\langle Y\rangle$ is well define. Now, we show $\varphi \circ \pi_{X}=\alpha \circ \pi_{Y}$. For any $r \in R$

$$
\begin{aligned}
\left(\varphi \circ \pi_{X}\right)(r) & =\varphi\left(\pi_{X}(r)\right) \\
& =\varphi(r+\langle X\rangle) \\
& =\alpha(r+\langle Y\rangle) \\
& =\alpha\left(\pi_{Y}(r)\right) \\
& =\left(\alpha \circ \pi_{Y}\right)(r)
\end{aligned}
$$

Corollary 2.9. If $\alpha$ is surjective then $R /\langle X\rangle \cong P /\langle Y\rangle$.

Proof. To prove $R /\langle X\rangle \cong P /\langle Y\rangle$, it will be shown that $\varphi$ is injective and surjective. Take any $r_{1}+\langle X\rangle, r_{2}+\langle X\rangle \in R /\langle X\rangle$ with $\varphi\left(r_{1}+\langle X\rangle\right)=\varphi\left(r_{2}+\langle X\rangle\right)$. We show $r_{1}+\langle X\rangle=$ $r_{2}+\langle X\rangle$ or $r_{1}-r_{2} \in\langle X\rangle$.

$$
\begin{aligned}
\varphi\left(r_{1}+\langle X\rangle\right) & =\varphi\left(r_{2}+\langle X\rangle\right) \\
\alpha\left(r_{1}\right)+\langle Y\rangle & =\alpha\left(r_{2}\right)+\langle Y\rangle \\
\alpha\left(r_{1}\right)-\alpha\left(r_{2}\right) & \in\langle Y\rangle \\
\alpha\left(r_{1}-r_{2}\right) & \in\langle Y\rangle
\end{aligned}
$$


It means that $r_{1}-r_{2}$ in $\langle X\rangle$. So $\varphi$ is injective. Next, take any $p+\langle Y\rangle \in P /\langle Y\rangle$. We show there exist $r+\langle X\rangle \in R /\langle X\rangle$ such that $\varphi(r+\langle X\rangle)=p+\langle Y\rangle$. We know that $p+\langle Y\rangle \in P /\langle Y\rangle$, it means there exist $p \in P$ such that $\pi_{Y}(p)=p+\langle Y\rangle$. Since $\alpha$ is surjective, can be found $r$ in $R$ such that $\alpha(r)=p$. Thus $\pi_{X}(r)=r+\langle X\rangle$, which $\varphi(r+\langle X\rangle)=p+\langle Y\rangle$. So $\varphi$ is surjective. Then $R /\langle X\rangle \cong P /\langle Y\rangle$.

\subsection{Module over a Non Associative Ring}

In the next discussion, we will define a module over a nonassociative ring.

Definition 2.10. Given $R$ is a non associative ring. Then $M$ is a module over a non-associative ring $R$ if it satisfies the following properties:

1. $(M,+)$ is a commutative group,

2. there is a multiplication $R \times M \rightarrow M ;(r, \mu) \mapsto r \mu$, for $r \in R$ and $\mu \in M$,

3. for $r_{1}, r_{2} \in R, \mu_{1}, \mu_{2} \in M$ satisfies the conditions:

(a) $r_{1}\left(\mu_{1}+\mu_{2}\right)=r_{1} \mu_{1}+r_{1} \mu_{2}$

(b) $\left(r_{1}+r_{2}\right) \mu_{1}=r_{1} \mu_{1}+r_{2} \mu_{1}$.

Now we give some examples of the module over a nonassociative ring.

Example 2.11. The set of real matrix $3 \times 1$ denoted by $M_{3 \times 1}(\mathbb{R})$ is a module over a non associative ring $\left(\mathbb{R}^{3},+, \times\right)$.

Example 2.12. The set of all derivation in $R_{J}$, denoted by $\operatorname{Der}\left(R_{J}\right)$, is an $R_{J}$-module, where $R_{J}$ is any Jordan ring (definition of derivations refers to [6]]).

Example 2.13. Given Hilbert space $\mathcal{H}$ and Jordan-Lie Algebra of operators in $\mathcal{H}$ (denoted by $\mathcal{A}_{\mathbb{R}}$ ). The Hilbert space $\mathcal{H}$ is an $\mathcal{A}_{\mathbb{R}}$-module with linear mapping

$$
\begin{gathered}
\mathcal{A}_{\mathbb{R}} \times \mathcal{H} \rightarrow \mathcal{H} \\
(\xi, \nu) \mapsto \xi(\nu)
\end{gathered}
$$

Then for all $\xi, \psi \in \mathcal{A}_{\mathbb{R}}$ and $\nu_{1}, \nu_{2} \in \mathcal{H}$, it is clear that $\xi\left(\nu_{1}+\nu_{2}\right)=\xi\left(\nu_{1}\right)+\xi\left(\nu_{2}\right)$ and $(\xi+\psi)\left(\nu_{1}\right)=\xi\left(\nu_{1}\right)+\psi\left(\nu_{1}\right)$. Therefore $\mathcal{H}$ is a module over a non associative ring $\mathcal{A}_{\mathbb{R}}$.

The unital axiom is satisfied if ring $R$ has a unit so that a unitary module will be formed as in the following definition.

Definition 2.14. If $R$ has a unit, $M$ is an R-module and for every $\mu$ in $M$ satisfies $1 \mu=\mu$, then $M$ is called an unital module over a non-associative ring.

In the previous section, we discussed the associative quotient ring $R /\langle X\rangle$. Thus we can construct the module over this ring.

Lemma 2.15. Let $R$ be a non-associative ring, $X=\{u(v w)-$ $(u v) w \mid u, v, w \in R\} \subset R$ and $M$ is a module over $R$. Then $M$ is a module over a quotient ring $R /\langle X\rangle$ if $\langle X\rangle$ is in the annihilator of $M$, denoted by $A n n_{R}(M)$.
Proof. Take arbitrary $r_{1}+\langle X\rangle, r_{2}+\langle X\rangle \in R /\langle X\rangle$ with $r_{1}+$ $\langle X\rangle=r_{2}+\langle X\rangle$ and $\mu \in M$. We will show that $\left(r_{1}+\langle X\rangle\right) \mu=$ $\left(r_{2}+\langle X\rangle\right) \mu$. It means that:

$$
\begin{aligned}
r_{1} \mu & =r_{2} \mu \\
r_{1} \mu-r_{2} \mu & =0 \\
\left(r_{1}-r_{2}\right) \mu & =0
\end{aligned}
$$

This condition will be satisfied if $\left(r_{1}-r_{2}\right) \in \operatorname{Ann}_{R}(M)$. So we get $r_{1}-r_{2} \in\langle X\rangle \subseteq A n n_{R}(M)$.

Furthermore, for a non-associative ring $R$ and an associative ring $S$ we obtain an $(R, S)$-bimodule. For a special case, we take $S=\operatorname{End}_{R}(R)$ which is associative.

If $M$ is left module over an associative ring $S$ and also right module over a non-associative ring $R$, then we cannot always construct a bimodule, since for all $s \in S, \mu \in M$, and $r \in R$, $(s \mu) r$ not always equal to $s(\mu r)$.

\subsection{Tensor Module Over Jordan Ring $R_{J}$}

In this section, we construct the tensor product on the modules over Jordan ring $R_{J}$. First, we define the mapping which is $R_{J}$-balanced.

Definition 2.16. Let $M_{1}, M_{2}$ be $R_{J}$-modules, $G$ be a commutative group, and $\lambda: M_{1} \times M_{2} \rightarrow G$ be a $\mathbb{Z}$-bilinear map. $A$ map $\lambda$ is called $R_{J}$-balanced if for all $\mu \in M_{1}, \nu \in M_{2}$, and $r \in R, \lambda(r \mu, \nu)=\lambda(\mu, r \nu)$.

Now we define the tensor product.

Definition 2.17. The tensor product $M_{1} \otimes_{R_{J}} M_{2}$ is an $R_{J}$-module together with a $R_{J}$-balanced map $\otimes$ : $M_{1} \times M_{2} \rightarrow M_{1} \otimes_{R_{J}} M_{2}$ where for every $\lambda: M_{1} \times$ $M_{2} \rightarrow G$ there is a unique linear map $\rho: M_{1} \otimes_{R_{J}}$ $M_{2} \rightarrow G$ such that $\lambda=\rho \circ \otimes$, like following diagram:

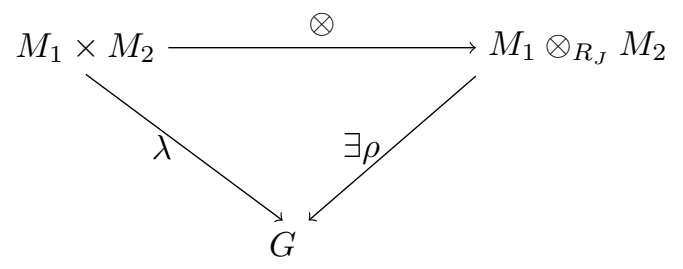

Consider the following explanation to show the existence of tensor product $M_{1} \otimes_{R_{J}} M_{2}$.

Lemma 2.18. The tensor product $M_{1} \otimes_{R_{J}} M_{2}$ exists.

Proof. Let $M_{1}$ and $M_{2}$ be modules over $R_{J}$. We can construct the direct sum like the following form:

$$
\mathbf{F}=\bigoplus_{M_{1} \times M_{2}} \mathbb{Z}_{(\mu, \nu)} \simeq \mathbb{Z}^{\left(M_{1} \times M_{2}\right)}
$$

This is a free module. From here, we find a basis in $\mathbf{F}$, i.e. $f_{(\mu, \nu)}=[\mu, \nu]$. If $N$ is a submodule in $\mathbf{F}$ generated by

$$
\begin{gathered}
{\left[\mu_{1}+\mu_{2}, \nu\right]-\left[\mu_{1}, \nu\right]-\left[\mu_{2}, \nu\right],} \\
{\left[\mu, \nu_{1}+\nu_{2}\right]-\left[\mu, \nu_{1}\right]-\left[\mu, \nu_{2}\right],} \\
{[r \circ \mu, \nu]-[\mu, r \circ \nu]}
\end{gathered}
$$


for $\mu, \mu_{1}, \mu_{2} \in M_{1}, \nu, \nu_{1}, \nu_{2} \in M_{2}, r \in R_{J}$. Choose $M_{1} \otimes_{R_{J}} M_{2}:=\mathbf{F} / N$, and define the map:

$$
\begin{aligned}
\tau: M_{1} \times M_{2} & \rightarrow M_{1} \otimes_{R_{J}} M_{2} \\
(\mu, \nu) & \mapsto \mu \otimes \nu:=[\mu, \nu]+N .
\end{aligned}
$$

From the definition of $N, \tau$ is $R_{J}$-balanced, not surjective, but image of $\tau$ is a generating set of $M_{1} \otimes_{R_{J}} M_{2}$ as a $\mathbb{Z}$-module. Since $\lambda$ is an $R_{J}$-balanced map from $M_{1} \times M_{2}$ to any grup $G$, we obtain a $\mathbb{Z}$-homomorphism $\rho: M_{1} \otimes_{R_{J}} M_{2} \rightarrow G,[\mu, \nu] \mapsto$ $\lambda(\mu, \nu)$ and $N \subset \operatorname{Ker}(\rho)$. Hence the following diagram is com-

mute:

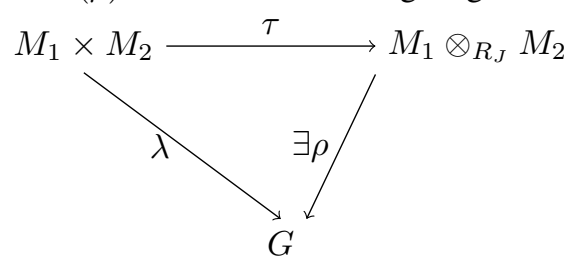

The map $\rho$ is unique. Then we can write the elements of $M_{1} \otimes_{R_{J}} M_{2}$ by

$$
\mu_{1} \otimes \nu_{1}+\ldots+\mu_{k} \otimes \nu_{k} .
$$

This form is not unique. Because $\mu \otimes \nu$ only represents a coset. So we get the map $\tau$ as a tensor product. With another word that tensor product $M_{1} \otimes_{R_{J}} M_{2}$ exists.

The following lemma shows that the tensor module over ring $R_{J}$ is an $R_{J}$-module.

Lemma 2.19. Let $M_{1}, M_{2}$ be $R_{J}$-modules. Then $M_{1} \otimes_{R_{J}}$ $M_{2}$ is an $R_{J}$-module.

Proof. To prove this lemma, we define additional and scalar multiplication in $M_{1} \otimes_{R_{J}} M_{2}$. Let $N$ be a submodule in $M_{1} \times M_{2}$. By definition $\otimes: M_{1} \times M_{2} \rightarrow M_{1} \otimes_{R} M_{2}$, with $(\mu, \nu) \mapsto \mu \otimes \nu:=[\mu, \nu]+N$. For all $\mu, \mu_{1} \in M_{1}, \nu, \nu_{1} \in M_{2}$, we obtain $\mu \otimes \nu+\mu_{1} \otimes \nu_{1}=[\mu, \nu]+N+\left[\mu_{1}, \nu_{1}\right]+N=$ $\left([\mu, \nu]+\left[\mu_{1}, \nu_{1}\right]\right)+N=\left[\mu+\mu_{1}, \nu+\nu_{1}\right]+N$. From this, we show that $M_{1} \otimes_{R_{J}} M_{2}$, is a commutative group. Then $\forall r \in R_{J}, \mu \in M_{1}, \nu \in M_{2}$, we define $r \circ(\mu \otimes \nu)=(r \circ \mu) \otimes \nu=\mu \otimes(r \circ \nu)$. According to Defintion 2.10 so $M_{1} \otimes_{R_{J}} M_{2}$ as $R_{J}$-module is proven.

Now, we know that $M_{1} \otimes_{R_{J}} M_{2}$ is an $R_{J}$-module. Following are the properties of the tensor product.

Lemma 2.20. The tensor product $M_{1} \otimes_{R_{J}} M_{2}$ is isomorphic to $M_{2} \otimes_{R_{J}} M_{1}$.

Proof. Construct the following diagram:

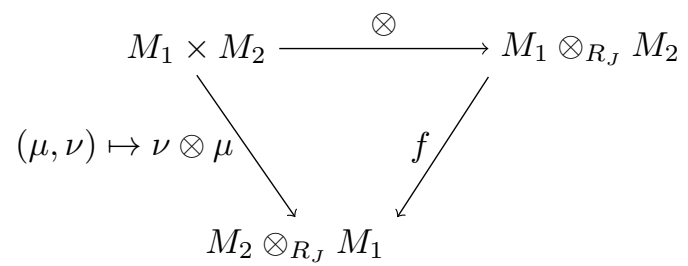

Then we define

$$
g: M_{2} \otimes_{R_{J}} M_{1} \rightarrow M_{1} \otimes_{R_{J}} M_{2}
$$

where $g(\nu \otimes \mu)=\mu \otimes \nu$. It will be proved that $f$ is an inverse of $g$ and vice versa. Let $a \in M_{2} \otimes_{R_{J}} M_{1}$ so by [7], $a$ spanning by $\nu \in M_{2}$ and $\mu \in M_{1}$, and we get

$$
\begin{aligned}
f(g(a)) & =f(g(\nu \otimes \mu)) \\
& =f(\mu \otimes \nu) \\
& =\nu \otimes \mu
\end{aligned}
$$

Analog for $g(f(a))$. Then we show that $f$ and $g$ are inverses of each other. It means that $M_{1} \otimes_{R_{J}} M_{2} \cong M_{2} \otimes_{R_{J}} M_{1}$.

Lemma 2.21. The tensor product $\left(M_{1} \otimes_{R_{J}} M_{2}\right) \otimes_{R_{J}} M_{3}$ is isomorpic to $M_{1} \otimes_{R_{J}}\left(M_{2} \otimes_{R_{J}} M_{3}\right)$.

Proof. Let $M_{1}, M_{2}$, and $M_{3}$ be $R_{J}$-module. We know that $(\mu \otimes \nu) \otimes \sigma$ is the linear generator of $\left(M_{1} \otimes_{R_{J}} M_{2}\right) \otimes_{R_{J}} M_{3}$, and $\mu \otimes(\nu \otimes \sigma)$ is the linear generator of $M_{1} \otimes_{R_{J}}\left(M_{2} \otimes_{R_{J}} M_{3}\right)$. To create the linear map

$$
\left(M_{1} \otimes_{R_{J}} M_{2}\right) \otimes_{R_{J}} M_{3} \rightarrow M_{1} \otimes_{R_{J}}\left(M_{2} \otimes_{R_{J}} M_{3}\right)
$$

with effect $(\mu \otimes \nu) \otimes \sigma \mapsto \mu \otimes(\nu \otimes \sigma)$, consider the function

$$
\begin{gathered}
M_{1} \times M_{2} \times M_{3} \rightarrow M_{1} \otimes_{R_{J}}\left(M_{2} \otimes_{R_{J}} M_{3}\right) \\
(\mu, \nu, \sigma) \mapsto \mu \otimes(\nu \otimes \sigma)
\end{gathered}
$$

Since $\mu \otimes(\nu \otimes \sigma)$ is trilinear in $\mu, \nu, \sigma$, we get a bilinear map

$$
\lambda_{\sigma}: M_{1} \times M_{2} \rightarrow M_{1} \otimes_{R_{J}}\left(M_{2} \otimes_{R_{J}} M_{3}\right)
$$

for each $\sigma$, with $\lambda_{\sigma}(\mu, \nu)=\mu \otimes(\nu \otimes \sigma)$, which induces a linear map

$$
f_{\sigma}: M_{1} \otimes_{R_{J}} M_{2} \rightarrow M_{1} \otimes_{R_{J}}\left(M_{2} \otimes_{R_{J}} M_{3}\right)
$$

such that $f_{\sigma}(\mu \otimes \nu)=\mu \otimes(\nu \otimes \sigma)$, for all elementary tensor $\mu \otimes \nu \in M_{1} \otimes_{R_{J}} M_{2}$. Next we show that map

$$
\begin{aligned}
\left(M_{1} \otimes_{R_{J}} M_{2}\right) \otimes_{R_{J}} M_{3} & \rightarrow M_{1} \otimes_{R_{J}}\left(M_{2} \otimes_{R_{J}} M_{3}\right) \\
(a, \sigma) & \mapsto f_{\sigma}(a) .
\end{aligned}
$$

is bilinear. First, the map is linear in $a$ which $\sigma$ fixed, because $f_{\sigma}$ is linear map. Then we will show that the map is linear in $\sigma$ which $a$ fixed. We show $f_{\sigma+\sigma^{\prime}}(a)=f_{\sigma}(a)+f_{\sigma^{\prime}}(a)$ and $f_{r \circ \sigma}(a)=r f_{\sigma}(a)$, where $a=\mu \otimes \nu$,

$$
\begin{aligned}
f_{\sigma+\sigma^{\prime}}(\mu \otimes \nu) & =(\mu \otimes \nu) \otimes\left(\sigma+\sigma^{\prime}\right) \\
& =(\mu \otimes \nu) \otimes \sigma+(\mu \otimes \nu) \otimes \sigma^{\prime} \\
& =f_{\sigma}(\mu \otimes \nu)+f_{\sigma^{\prime}}(\mu+\nu) \\
& =\left(f_{\sigma}+f_{\sigma^{\prime}}\right)(\mu \otimes \nu) .
\end{aligned}
$$

Then $f_{r \circ \sigma}(\mu \otimes \sigma)=(\mu \otimes \nu) \otimes(r \circ \sigma)=r \circ((\mu \otimes \nu) \otimes \sigma)$. So $f_{\sigma}(a)$ is linear in $\sigma$ and $a$. By the universal mapping properties (UMP) of tensor product, there is a unique linear map

$$
f:\left(M_{1} \otimes_{R_{J}} M_{2}\right) \otimes_{R_{J}} M_{3} \rightarrow M_{1} \otimes_{R_{J}}\left(M_{2} \otimes_{R_{J}} M_{3}\right)
$$

such that $f(a \otimes \sigma)=f_{\sigma}(a)$. So we get $f((\mu \otimes \nu) \otimes \sigma)=$ $f_{\sigma}(\mu \otimes \nu)=\mu \otimes(\nu \otimes \sigma)$. Analog to another map

$$
g: M_{1} \otimes_{R_{J}}\left(M_{2} \otimes_{R_{J}} M_{3}\right) \rightarrow\left(M_{1} \otimes_{R_{J}} M_{2}\right) \otimes_{R_{J}} M_{3}
$$


where $g(\mu \otimes(\nu \otimes \sigma))=(\mu \otimes \nu) \otimes \sigma$. In next step, will be shown that $f$ is invers of $g$ and $g$ is invers of $f$, i.e.

$$
\begin{aligned}
g(f((\mu \otimes \nu) \otimes \sigma) & =g\left(f_{\sigma}(\mu \otimes \nu)\right) \\
& =g(\mu \otimes(\nu \otimes \sigma)) \\
& =(\mu \otimes \nu) \otimes \sigma
\end{aligned}
$$

and

$$
\begin{aligned}
f(g(\mu \otimes(\nu \otimes \sigma) & =f\left(g_{\mu}(\nu \otimes \sigma)\right) \\
& =f((\mu \otimes \nu) \otimes \sigma)) \\
& =(\mu \otimes(\nu \otimes \sigma) .
\end{aligned}
$$

From Lemma 2.21, we know that the tensor product of the module is associative up to isomorphism but not by element. Consider the following illustration. For all $r_{1}, r_{2}, r_{3} \in R_{J}, \mu \in$ $M_{1}, \nu \in M_{2}$, dan $\sigma \in M_{3}$,

$$
\begin{aligned}
& \left(\left(r_{1} \circ \mu\right) \otimes\left(r_{2} \circ \nu\right)\right) \otimes\left(r_{3} \circ \sigma\right) \\
= & \left(\left(r_{1} \circ r_{2}\right) \circ(\mu \otimes \nu)\right) \otimes\left(r_{3} \circ \sigma\right) \\
= & \left(\left(r_{1} \circ r_{2}\right) \circ r_{3}\right) \circ((\mu \otimes \nu) \otimes \sigma) \\
\neq & \left(r_{1} \circ\left(r_{2} \circ r_{3}\right)\right) \circ((\mu \otimes \nu) \otimes \sigma)
\end{aligned}
$$

It means $\left(\left(r_{1} \circ \mu\right) \otimes\left(r_{2} \circ \nu\right)\right) \otimes\left(r_{3} \circ \sigma\right) \neq\left(r_{1} \circ \mu\right) \otimes\left(\left(r_{2} \circ\right.\right.$ $\left.\nu) \otimes\left(r_{3} \circ \sigma\right)\right)$.

\section{Potential Applications}

In the previous section, we defined the modules a over nonassociative ring. If an inner product is defined in such modules, a so-called pre-Hilbert module will be obtained. If the preHilbert module is complete with respect to the norm defined by the inner product, it is called a Hilbert module over a nonassociative ring .

Quantum mechanics is one of the fundamental theories of physics that describes microscopic physical systems. According to quantum mechanics, every quantum system corresponds to a separable extended Hilbert space. In [8, 9], the Hilbert space is replaced with Hilbert module over the ring of all quaternions. Motivated by such extension of the mathematical formulation of quantum mechanics, i.e., by replacing the Hilbert space with the quaternionic Hilbert module as the state space of quantum mechanics, we will formulate in the next article another possible extension of quantum mechanics by replacing the Hilbert space with a Hilbert module over a nonassociative ring and investigate the implications of such replacement.

\section{Conclusions}

Based on the result, we construct a non associative ring from any ring $R$, denoted by $R_{J}$. All of ideals in non-associative ring are non associative, except ideal that generate by $X=$ $\{u(v w)-(u v) w \mid u, v, w \in R\} \subset \operatorname{Ann}_{R}(R)$.

We define the module over a non-associative ring. Let $S$ be an associative ring, and $R$ be a non-associative ring. If $M$ is the left module over $S$ and also as the right module over $R$, then we cannot always construct a bimodule. We also construct the tensor modules over a non-associative ring. The tensor module is commutative and associative up to isomorphism, but not element by element.

\section{Acknowledgements}

The authors also thanks referees for useful comments and suggestions.

\section{REFERENCES}

[1] T. Shah, A. Razzaque, I. Rehman, M.A. Gondal, and K.P. Shum, Literature Survey on Non-Associative Rings and Development, Europeon Journal of Pure And Aplied Mathematics, Vol. 12. No 2, 370-408, 2019.

[2] A. Razzaque, Dissertation: Investigation og a Class of NonAssociative Ring and Its Study using Soft Set, Department of Mathematics Quaid-i-Azam University, Islamabad, Pakistan, 2016.

[3] A. Carotenuto, Connection on Jordan Modules and Particle Physics, Conference: Corfu Summer Institute 2017 "Schools and Workshops on Elementary Particle Physics and Gravity", Carfu, Greece, 2017.

[4] A. Carotenuto, L. Dabrowski, M. Dubois-Violette, Differential Calculus on Jordan Algebra and Jordan Modules, Letter in Mathematical Physics, No.109, 113-133, 2019.

[5] N.P. Landsman, Mathematical Topics Between Classical and Quantum Mechanics, Springer-Verlag New York, Inc. 1998.

[6] M. Ashraf, S. Ali, and C. Haetinger, On Derivations in Rings and Their Applications, The Aligarh Bulletin of Mathematics, Vol 25, No. 2, page 79-107, 2006

[7] K. Conrad, Tensor Product, Online available from https://kconrad.math.uconn.edu/blurbs. 2016.

[8] A.I. Arbab, The Quaternion Quantum Mechanics, Applied Physics Research, Vol. 3, No. 2, page 160-170, 2011.

[9] M. Danielewski and L. Sapa, Foundations of the Quaternion Quantum Mechanics, Entropy, Vol.22(12)1424, 2020. 